\title{
Protective Effect of Polysaccharide Fractions from Radix A. Sinensis against tert-Butylhydroperoxide Induced Oxidative Injury in Murine Peritoneal Macrophages
}

\author{
Xingbin Yang ${ }^{1, *}$, Yan Zhao', You Lv', Ying Yang ${ }^{1}$ and Yun Ruan' \\ ${ }^{1}$ Key Laboratory of Ministry of Education for Medicinal Plant Resource and Natural Pharmaceutical Chemistry, \\ College of Life Sciences, Shaanxi Normal University, Xi' an 710062, China \\ ${ }^{2}$ Faculty of Pharmaceutical Sciences, Fourth Military Medical University, Xi'an 710032, China
}

Received 30 April 2007, Accepted 6 July 2007

\begin{abstract}
Three Angelica sinensis polysaccharide fractions (APFs), named APF1, APF2 and APF3, were isolated and purified from Radix $A$. sinensis and their antioxidant activities were evaluated in isolated mouse peritoneal macrophages by pretreatment with APFs before exposure to $0.2 \mathrm{mM}$ tertbutylhydroperoxide ( $t$-BHP). The results showed that pretreatment of the macrophages with APFs as low as $\mathbf{1 0}$ $\mu \mathrm{g} / \mathrm{ml}$ could significantly enhance $t$-BHP-decreased cell survival, intracellular glutathione (GSH) content and superoxide dismutase (SOD) activity, and also inhibited $t$ BHP-increased lactate dehydrogenase (LDH) leakage and malondialdehyde (MDA) formation $(p<0.05)$, and APF3 was the most active fraction, followed by APF2 and APF1 in decreasing order. Furthermore, we found for the first time that the bound-protein in APF3 was associated closely with the protective effects and the polysaccharide inhibited the excess NO release from $t$-BHP-activated macrophages to protect host cells.
\end{abstract}

Keywords: Antioxidant activity, Macrophages, Polysaccharide fractions, Radix A. sinensis, Tert-Butylhydroperoxide

\section{Introduction}

In recent years, natural polysaccharides as an important biopolymer mainly from plenty of plants source have been shown to exhibit various biological activities, such as antitumour, immunomodulatory, anticancer, anti-inflammatory, anticoagulant hypoglycaemic and antiviral activities (Abe et al., 1983; Srivastava and Kulshreshtha, 1989; Shahidi and Synowiecki,

*To whom correspondence should be addressed.

Tel: 86-29-85310266; Fax: 86-29-85303736

E-mail: xbyang@snnu.edu.cn.
1991; Majeti and Ravi Kumar, 2000; Fang et al., 2006; Jeong et al., 2006). Therefore, it is of interest to discover and evaluate biopolymeric polysaccharide fractions as new safe plant-based health foods and drugs.

In thousands of years, Radix Angelica sinensis (Umbelliferae family) has been widely used as a common food and folk medicine in several Asian countries because of its broad pharmacological effects (Zhao et al., 2003; Lao et al., 2004; Lu et al., 2004; Kim et al., 2005; Deng et al., 2006). In recent years, the pharmaceutical products, especially the polysaccharide biopolymers isolated from the Radix A. sinensis have received extensive attention. However, the studies on the polysaccharides have mainly focused on immunomodulatory activity (Sun et $a l ., 2005)$ and until now, there is relatively little information with respect to the antioxidant activity.

It is well known that exposure of organisms to exogenous and endogenous factors usually generates a wide range of reactive oxygen species (ROS), resulting in the homeostatic imbalance and adverse effects on human health (BonnefontRousselot et al., 2000; Kim and Kang, 2006; Mansour et al., 2006). Therefore, the studies on protective effect of plant polysaccharides against oxidative stress have become a subject of considerable interest. For searching for new antioxidants, the present study was designed to assess the antioxidant ability of polysaccharide fractions from Radix $A$. sinensis against tertbutylhydroperoxide ( $t$-BHP)-mediated oxidative stress in murine peritoneal macrophages in vitro.

\section{Materials and Methods}

Reagents. Triton X-100 was obtained from Amersco Inc. (Solon, OH, USA). RPMI1640, phosphate buffer saline (PBS) and fetal bovine serum (FBS) were the product of Gibco BRL. tert-Butylhydroperoxide ( $t$-BHP), dimethyl sulfoxide (DMSO), 3-[4,5-dimethylthiazole-2-yl]2,5-diphenyltetrazoliumbromide (MTT), polymyxin B (PMB) and 
lipopolysaccharide (LPS) were purchased from Sigma. All other chemicals and solvents were of analytical grade.

Isolation and chemical characterization of polysaccharide fractions. Radix A. sinensis were purchased from Minxian County, Gansu Province, China and were identified according to the identification standard of Pharmacopeia of the People's Republic of China. The dried Radix A. sinensis (550 g) were defatted with $95 \%$ alcohol and then extracted with distilled water $(\mathrm{g} / \mathrm{ml}=1: 10)$ for $3 \mathrm{~h}$. After each three-hour period of water extracting, the water extracts were collected and the residue was extracted again for 3 cycles. The combined extracts were pooled and concentrated to $30 \%$ of the original volume under a reduced pressure and then centrifuged at 3,000 rpm for $15 \mathrm{~min}$. The supernatant was collected and three volume of $95 \%$ alcohol was added slowly and stirred to precipitate the polysaccharides, and then the mixture was stored overnight at $4^{\circ} \mathrm{C}$ and finally the polysaccharide pellets were obtained by centrifugation at $4,000 \mathrm{rpm}$ for $15 \mathrm{~min}$. The polysaccharide pellets were completely dissolved in appropriate volume of distilled water and intensively dialyzed for 2 days against distilled water (cut-off $\mathrm{M}_{\mathrm{w}} 8000 \mathrm{Da}$ ). The retentate portion was concentrated, deproteinated by freeze-thaw process (BenchTOP, Virtis Co. USA) for repeating seven times and centrifuged to remove insoluble material. Finally the supernatant was lyophilized and the resulting amorphous powder was named as crude biopolymeric polysaccharide fractions from Radix A. sinensis. Furthermore, one gram of the crude polysaccharide fractions was dissolved in $0.1 \mathrm{M} \mathrm{NaCl}$ solution and filtered through a filter paper $(0.45 \mu \mathrm{m})$. Then the resolution was applied to Sephacryl S-400 gel filtration column chromatography and eluted with $0.1 \mathrm{M} \mathrm{NaCl}$ solution. Eluted each solution $(5 \mathrm{ml})$ was collected and monitored by sugar content based on phenol-sulfuric acid method at $490 \mathrm{~nm}$. Finally, the eluted solution was concentrated and lyophilized to yield three white Radix A. sinensis polysaccharide fractions (APFs), namely APF1, APF2 and APF3 with different molecular size according to the elution profile.

Moreover, component monosaccharides in AFPs were separated and quantified by HPLC method as our previously described (Yang et al., 2005). The obtained AFPs are white powder readily soluble in water and the predominant sugars in various APFs differed from one polysaccharide fraction to another. APF1 consisted of rhamnose, galacturonic acid, glucose, galactose and arabinose with the molar ratio of $1.00: 2.65: 2.02: 3.45: 10.64$; APF2 consisted of mannose, rhamnose, galacturonic acid, glucose, galactose and arabinose with the molar ratio of $0.44: 1.00: 10.52: 7.52: 8.19$ : 14.43; APF3 consisted of mannose, rhamnose, glucuronic acid, galacturonic acid, glucose, galactose and arabinose with the molar ratio of $0.74: 1.00: 0.25: 9.06: 8.62: 5.94: 9.28$.

Macrophage isolation, culture and drug treatments. Macrophages were prepared from BALB/c mice (6-8 weeks old, 17-20 g body weight) as described previously (Kim et al., 2004). Briefly, peritoneal macrophages were harvested from $3 \mathrm{BALB} / \mathrm{c}$ mice. The collected cells were seeded and cultured in RPMI 1640 containing 10\% heatinactivated FBS, $100 \mathrm{U} / \mathrm{ml}$ penicillin and $100 \mu \mathrm{g} / \mathrm{ml}$ streptomycin at a density $2 \times 10^{6}$ cells/well. The cells were allowed to adhere for $3 \mathrm{~h}$ to a $96-w e l l$ culture plate at $37^{\circ} \mathrm{C}$ in a $5 \% \mathrm{CO}_{2}$ incubator. Then the cultures were washed twice with RPMI1640 to remove nonadherent cells prior to the addition of $1 \mathrm{ml}$ of fresh RPMI 1640 containing $10 \%$ FBS.

Stock solutions of $5.0 \mathrm{mg} / \mathrm{ml}$ AFPs were prepared in freshly PBS buffer ( $\mathrm{pH}$ 7.2) and added into RPMI 1640 medium to achieve various designed concentrations. The adherent macrophages were pre-treated with or without AFPs at the concentration of 10, 100, $500 \mu \mathrm{g} / \mathrm{ml}$ for $24 \mathrm{~h}$ before exposed to $0.2 \mathrm{mM} t$-BHP. The control group received vehicle only and there were five repetitions for each treatment. At the end of the designated treatment the cultured media were quickly collected, frozen and stored at $-20^{\circ} \mathrm{C}$ or immediately used for analyzing the release of extracellular lactate dehydrogenase (LDH) and nitric oxide (NO). Furthermore, the cultured cells were quickly harvested and washed with ice-cold phosphate-buffered saline (PBS, $0.1 \mathrm{M}, \mathrm{pH}=7.4$ ) and centrifuged at $1,000 \times g$ to pellet cells. Cells were then suspended in an appropriate volume of buffer (50 mM Tris- $\mathrm{HCl}$ pH 8.0, $50 \mathrm{mM}$ EDTANa $_{2}, 0.2 \mathrm{M} \mathrm{NaCl}, 1 \%$ Triton X-100) and subjected to four freeze-thaw cycles to lyse cells and the yielded cell homogenate was immediately centrifuged at $4^{\circ} \mathrm{C}$ for $20 \mathrm{~min}$ at $10,000 \times \mathrm{g}$. The afforded supernatant was then divided into several tubes and stored at $-20^{\circ} \mathrm{C}$ prior to the assays of malondialdehyde (MDA) centents, glutathione (GSH) levels and superoxide dismutase (SOD) activity.

Cell viability and $\mathrm{LDH}$ release assay. Macrophages were pretreated with the indicated concentration of AFPs, and then exposed to $t$ BHP as described above. Colorimetric MTT assay was performed to assess cell viability (Mossman, 1983; Carmichael et al., 1987). Briefly, $20 \mu \mathrm{l}$ of MTT $(5 \mathrm{mg} / \mathrm{ml})$ in PBS solution was added to each well at a final concentration of $5 \mathrm{mg} / \mathrm{ml}$ and then the plate was further incubated for $4 \mathrm{~h}$. All remaining supernatant were removed and $100 \mu \mathrm{l}$ of DMSO was added to each well and mixed thoroughly to dissolve the formed crystal formazan. After $10 \mathrm{~min}$ of incubation to ensure all crystals were dissolved, the light absorption was measured at $570 \mathrm{~nm}$ using an enzyme-linked immunosorbent assay (ELISA) reader. Viability was expressed as a percentage of absorbance values in treated cells to that in control cells.

The leakage into the media of $\mathrm{LDH}$, an indicator of cell injury, was detected with an assay kit (Jiancheng BioEngineering, Nanjing, China) according to the manufacturer's instructions as described by Mi and Zhang. (2005). Briefly, at the end of the incubation $30 \mu \mathrm{l}$ of cell medium was taken out for the activity analysis of extracellular $\mathrm{LDH}$, which could catalyze the conversion of lactate to pyruvate, and then reacted with 2,4-dinitrophenylhydrazine to give the brownish red color in basic solution. After reaction, each sample was detected and the absorbance was read at wavelength $440 \mathrm{~nm}$ and the results were also expressed as the percentage of $\mathrm{LDH}$ leakage versus control cells.

Measurement of NO production. NO production was determined indirectly by assaying the culture supernatant for accumulated nitrite, the stable end product of NO reacted with molecular oxygen as previously described (Green et al., 1982). Briefly, murine peritoneal exudate was plated into 96-well plate and adhered macrophages at $2 \times 10^{6}$ cells/well were cultured with various concentrations of polysaccharide fractions at $37^{\circ} \mathrm{C}$ for $24 \mathrm{~h}$. After treatment, $100 \mu \mathrm{l}$ of isolated supernatants were allowed to react with Griess reagent ( $1 \%$ sulfanilamide, $0.1 \% N$-1-naphthylethylenediamine dihydrochloride and $2.5 \%$ phosphoric acid) at room 
temperature for $10 \mathrm{~min}$. Nitrite products in cell supernatants were determined by measuring absorbance at $550 \mathrm{~nm}$ versus a $\mathrm{NaNO}_{2}$ standard curve using an ELISA reader and the results were shown as $\mu \mathrm{M}$.

Lipid peroxidation assay. MDA is a breakdown product of the oxidative degradation of cell membrane lipids and is generally considered an indicator of lipid peroxidation. In the present study, lipid peroxidation was thus evaluated by measuring MDA concentrations according to the thiobarbituric acid (TBA) method with a commercial kit (Jiancheng BioEngineering) as described previously (Uchiyama and Mihara, 1978). The method was based on the spectrophotometric measurement of the color produced during the reaction to TBA with MDA. MDA concentrations were calculated by the absorbance of TBA reactive substances (TBARS) at $532 \mathrm{~nm}$ and were expressed in nmol/ $/ 10^{6}$ cells.

Estimation of antioxidant activity in $\boldsymbol{t}$-BHP-induced macrophages. The SOD activity was examined according to xanthine oxidase method provided by a standard assay kit (Jiancheng BioEngineering) with minor modifications (Sun et al., 1988). The assay used the xanthine-xanthine oxidase system to produce superoxide anions, which react with 2-(4-iodophenlyl)-3-(4-nitrophenol-5-phenlyltetrazoliom chloride) to form a red formazan dye and the absorbance at $550 \mathrm{~nm}$ was determined. The values were expressed as units per $10^{6}$ cells, where one unit of SOD was defined as the amount of SOD inhibiting the rate of reaction by $50 \%$ at $25^{\circ} \mathrm{C}$.

Intracellular reduced GSH was determined by an Assay Kit (Jiancheng BioEngineering, China) as described previously (Van den Berg et al., 1992; Han et al., 2004). Briefly, The assay was carried out via an enzymatic recycling reaction of GSH in combination with a chromogenic reaction with 5,5'-dithio-bis-2nitrobenzoic acid (DTNB) in mixture contained $30 \mu \mathrm{l}$ of cell supernatants, $200 \mu \mathrm{l}$ PBS buffer (150 mM, pH 7.7) and $20 \mu \mathrm{l} 1-$ dithio-bis-2-nitrobenzoic acid (DTNB) in a total volume of $250 \mu \mathrm{l}$. After incubation at $30^{\circ} \mathrm{C}$ for $5 \mathrm{~min}$, this reaction finally led to the formation of 5-thio-2-nitrobenzoate (TNB) with the yellow color and the absorption maximum at $412 \mathrm{~nm}$ was measured at $412 \mathrm{~nm}$ against the blank on an multiwell plate reader. The GSH contents were determined from standard curves of known concentrations of total GSH and the GSH level was expressed as nmol/ $10^{6}$ cells.

Statistical Analysis. Data are expressed as mean and standard deviation (mean \pm S.D.) of the results obtained from the average of 5 independent experiments. Differences between two different groups were compared with the Student's $t$-tests and considered as statistically significant when the $p$ value was less than 0.05 .

\section{Results}

Effect of APFs on the viability of macrophages treated with $t$-BHP. In this study, cell viability was determined by MTT assay. Various concentrations of $t$-BHP $(100-500 \mu \mathrm{M})$ were tested to determine the optimal dose. The results showed that the relative cell survival of macrophage decreased in response to treatment with the $t$-BHP for $24 \mathrm{~h}$, with an

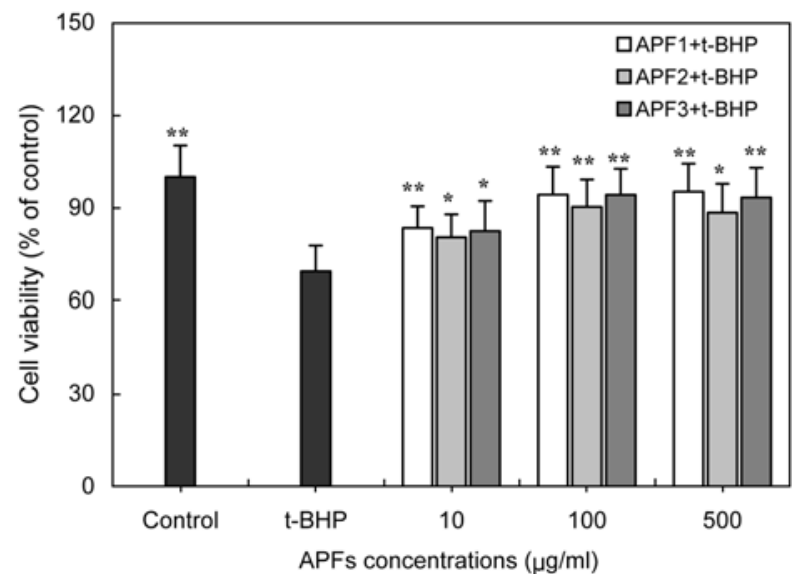

Fig. 1. Effect of biopolymeric polysaccharide fractions on $t$-BHPinduced cytotoxicity in mouse peritoneal macrophages. Cells at the concentration of $2 \times 10^{6}$ cells/well were pre-incubated with varying concentrations of APFs for $24 \mathrm{~h}$ and then were incubated with $0.2 \mathrm{mM} t$-BHP for another $24 \mathrm{~h}$. Cell viability was assessed by MTT reduction assay and expressed as the percentage of absorbance values to the control group. The cells in control group were cultured in the RPMI1640 medium and the cells in $0.2 \mathrm{mM} t$ BHP-treated alone were used as positive group. Values represent means \pm S.D. of five independent experiments. A difference was considered statistically significant when $* p<0.05$ and $* * p<0.01$ against only $t$-BHP-exposed group.

average $\mathrm{IC}_{50}$ value of about $250 \mu \mathrm{M}$ (data not shown). In a similar fashion, the effect of the polysaccharide fractions at a concentration range of $10-500 \mu \mathrm{g} / \mathrm{ml}$ on the survival of $t$ BHP-treated macrophages was also measured. As shown in Fig. 1, the addition of $0.2 \mathrm{mM} t$-BHP to the cells resulted in a significant increase in cytotoxicity, as revealed by a $30.6 \%$ fall in cell viability compared to control cells and the pretreatments with low dose of APFs $(10 \mu \mathrm{g} / \mathrm{ml})$ for $24 \mathrm{~h}$ significantly improved the cell viability by $14.0 \%$ for APF $1,11.0 \%$ for APF2 and $13.1 \%$ for APF3 compared to $t$-BHP-treated cells $(p<0.05)$, respectively. However, no significant differences in the protective effects were observed among three polysaccharide fractions. It was also found that when APFs up to $100 \mu \mathrm{g} / \mathrm{ml}$, over $90.3 \%$ viability was maintained through the test period. The results showed the APFs could protect the macrophages from $t$-BHP-induced cytotoxicity in vitro, indicating that APFs could act as the new antioxidants.

Assessment of cytotoxicity by $\mathrm{LDH}$ release assay. LDH release into the media was used as an index of the integrity of cell membranes or necrosis in response to the oxidant burden (Mi and Zhang, 2005). In this study, the cytotoxicity of $t$-BHP to cultured macrophages and the protective effect of APFs against the cellular injury were evaluated based on the $\mathrm{LDH}$ leakage. As shown in Fig. 2, there was a marked increase of LDH leakage by about 2.7-fold of the control $(5.8 \pm 0.4 \%$ versus $15.5 \pm 1.3 \%, p<0.01)$ after addition of $t$-BHP to the 


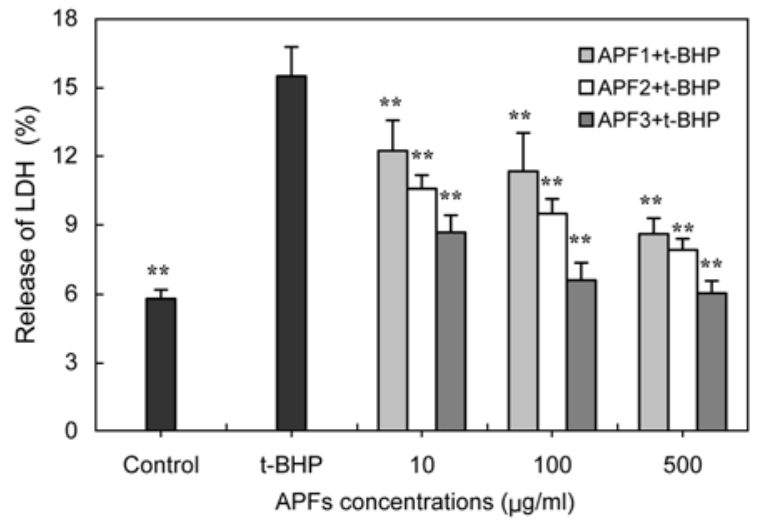

Fig. 2. Assessments of cytotoxicity by LDH leakage into medium from macrophages pretreated with indicated concentration of APFs. The cells were pre-incubated with various concentrations of APFs for $24 \mathrm{~h}$ before exposure to $t$-BHP. The medium was collected to assess LDH release and the percentage of LDH leakage was calculated to reflect the cytotoxicity as described in Methods. Data are presented as means \pm S.D. $(\mathrm{n}=5)$. $* * p<0.01$ indicates significant different versus only $t$-BHP-treated group.

culture media. However, this increase was markedly attenuated in a dose-dependent manner by the pre-incubation of macrophages with APFs in the range from 10 to $500 \mu \mathrm{g} / \mathrm{ml}$ for $24 \mathrm{~h}$ (Fig. 2), where extracellular LDH activity were reduced by $21.1 \%$ for $\mathrm{APF} 1,31.8 \%$ for APF2 and $44.1 \%$ for APF3 at the concentrations as low as $10 \mu \mathrm{g} / \mathrm{ml}(p<0.01)$, respectively. When pre-incubation with APFs at high concentration of $500 \mu \mathrm{g} / \mathrm{ml}, t$-BHP-induced LDH leakage were inhibited by $44.5 \%$ for APF1, $49.1 \%$ for APF2 and $61.1 \%$ for APF3 $(p<0.01)$, respectively. In the present study, the highest inhibitory activity was exhibited by APF3, followed by APF2 and APF1 (Fig. 2). Unlike the similar effect of APFs on the cell viability in MTT assays, the protective effects of the polysaccharide fractions on $t$-BHPinduced cytotoxicity differed from one polysaccharide fraction to another.

Measurement of oxidative damage by MDA formation. The toxicity caused by ROS was normally accompanied with the increase of lipid peroxides (Dipti et al., 2006). In the present study the oxidative damage was evaluated by the formation of MDA, a well-known biomarker of overall oxidative damage to cellular constituents such as membrane lipids. From the results illustrated in Fig. 3, it was found that the intracellular MDA production significantly increased after macrophages were exposed to $0.2 \mathrm{mM} t$-BHP and the MDA formation was dose-dependently reduced when the cells were pre-incubated with APFs at designed concentration range of $10-500 \mu \mathrm{g} / \mathrm{ml}$ for $24 \mathrm{~h}(p<0.05)$. Meanwhile, the MDA level in $t$-BHP-treated group was increased about 2.6-fold compared to the control cells, whereas the pretreatment of the cells with the polysaccharide fractions at concentrations of 10, 100 and $500 \mu \mathrm{g} / \mathrm{ml}$ could reduce over $17.2,22.1$ and $25.4 \%$ of $t$-BHP-

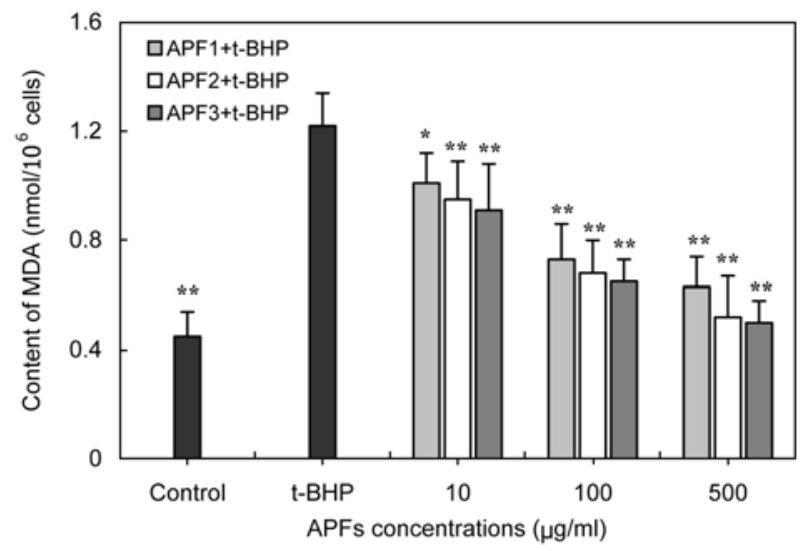

Fig. 3. Inhibitory effect of APFs on $t$-BHP-induced changes of MDA formation in macrophages. The cultured macrophages were pre-incubated with different concentrations of APFs for $24 \mathrm{~h}$ at $37^{\circ} \mathrm{C}$ and then were subjected to oxidative damage by $0.2 \mathrm{mM} t$ BHP. The cells were collected and the level of lipid peroxidation was evaluated by measuring intracellular MDA content. MDA content was calculated as $\mathrm{nmol} / 1 \times 10^{6}$ cells. ${ }^{*} p<0.05, \quad{ }^{*} p<0.01$ compared with only $t$-BHP-treated group.

induced MDA production, respectively. Especially, the pretreatment with $500 \mu \mathrm{g} / \mathrm{ml}$ APF2 or APF3 almost completely inhibited $t$-BHP-induced MDA formation and the highest activity was achieved by APF3, followed by APF2 and APF1 in decreasing order (Fig. 3). The results indicated that there was a significant increase in lipid peroxidation when the macrophages were exposed to $t$-BHP and the polysaccharide fractions did exhibit expectedly protective effect against the oxidative damage.

Inhibitory effects of $\boldsymbol{t}$-BHP-mediated SOD reduction and GSH depletion in macrophages. To further understand the antioxidant action of APFs, the effects of APFs on the SOD activity and GSH content were investigated in $t$-BHP-induced macrophages. As shown in Fig. 4A and B, after the cells were exposed to $0.2 \mathrm{mM} t$-BHP for $24 \mathrm{~h}$, intracellular GSH was depleted about $61.8 \%(p<0.01)$ and intracellular SOD activity was decreased by about $52.6 \%(p<0.01)$. However, the preprotection with various concentrations of APFs, a significant increase in GSH level and SOD activity were observed as low as $10 \mu \mathrm{g} / \mathrm{ml}$ and APFs attenuated the changes of $t$-BHPmediated SOD reduction and GSH depletion from macrophages in a dose-dependent manner ( $p<0.05$, Fig. $4 \mathrm{~A}$ and B). It was also found that APF3 exhibited higher activity than the other two fractions. After pre-incubated with APF3 at concentrations of 10,100 and $500 \mu \mathrm{g} / \mathrm{ml}$, GSH levels were recovered to 47.1 , 78.8 and $90.3 \%$, respectively and the pre-incubation also rescued the $t$-BHP-decreased SOD activity by 46.3, 63.5 and $88.8 \%$, respectively. As a result, it was further conformed that all the polysaccharide fractions possessed the significant antioxidant properties. 

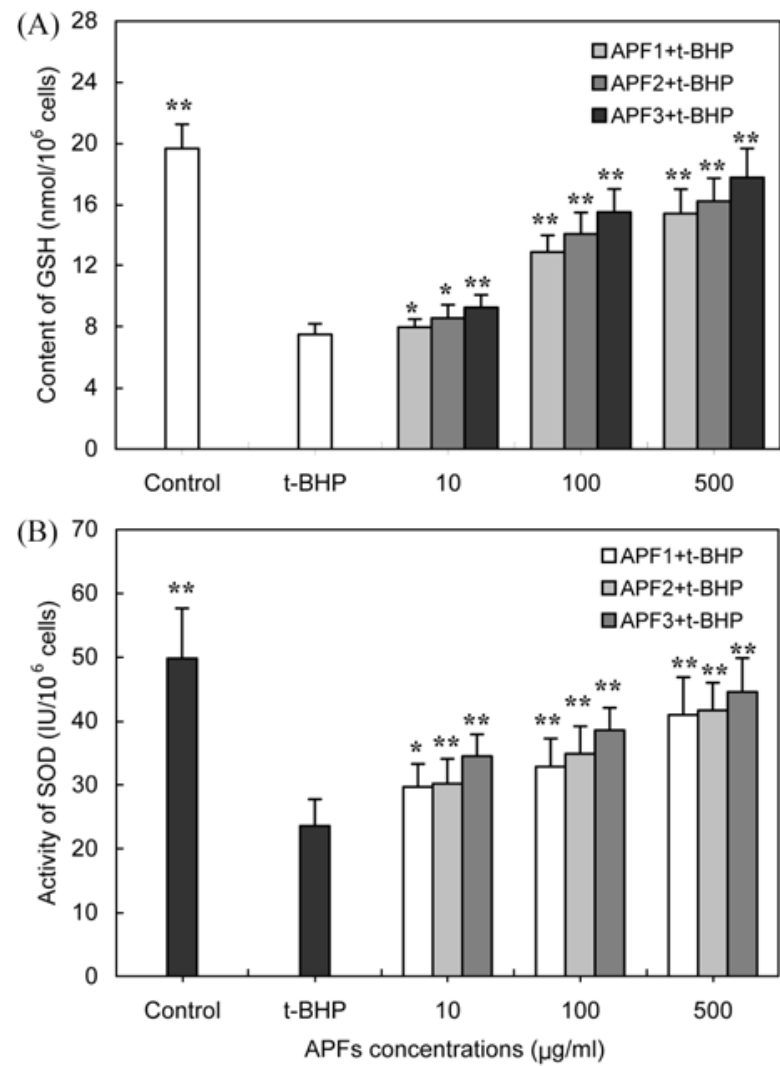

Fig. 4. Assessments of cytoprotective effect of APFs against $t$ BHP-induced oxidative damage in cultured macrophages. GSH level (A) and SOD activity (B) were assayed as described in Methods. Values are displayed as the means \pm S.D. $(n=5)$. ${ }^{*} p<0.05,{ }^{* *} p<0.01$ compared with only $t$-BHP-treated group.

Modulation of APFs on NO release from $\boldsymbol{t}$-BHP-induced macrophages. Although APFs have been shown to have markedly antioxidant activity in macrophages, it is unclear if APFs affected the formation of NO, a key ROS and function molecule released from activated macrophages (Rojas et al., 1993; MacMicking et al., 1997). Therefore, NO production was assessed by adding the high active APF3 (100 or $500 \mu \mathrm{g} /$ $\mathrm{ml}$ ) to a graded concentration of $t$-BHP-activated macrophages and the results were presented in Fig. 5. Interestingly, it was found that APFs accelerated NO release from the macrophages stimulated by $t$-BHP at low concentrations of 0.2 and $0.4 \mathrm{mM}$. As the concentrations of $t$-BHP up to $0.8 \mathrm{mM}$, an inhibitory effect of APFs on $t$-BHP-induced NO production in the cultured macrophages were observed and a further increase in $t$-BHP concentrations caused a significant inhibition of NO release by APF3 in macrophages $(p<0.05)$. For the first time we found that APF3 could inhibit NO release from high concentration of $t$-BHP-activated macrophages and the bifunctional effect strongly depended on the NO concentration in activated macrophages. Based on investigations, it was suggested that APFs exhibited dual function in NO production, which possibly protected host cells from the harm of excessive NO generation

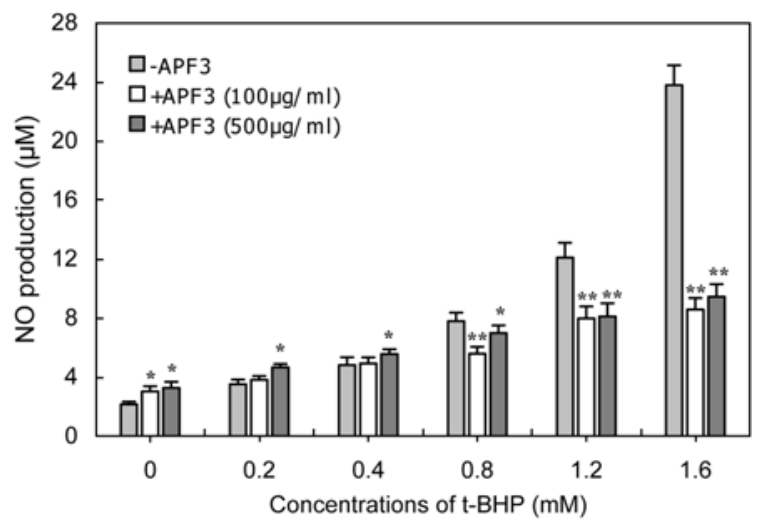

Fig. 5. Modulatory effects of APFs on NO production in $t$-BHPtreated macrophages. Cells were co-incubated with various concentration of $t$-BHP in the absence or presence of APF3. Date represent means $\pm \mathrm{SD}$ of 5 separate samples. ${ }^{*} p<0.05,{ }^{* *} p<0.01$ compared with only $t$-BHP-treated groups.

Effect of bound-protein in APF3 on the antioxidant activity. In this study, the co-extracted proteins in the polysaccharides were checked according to Lowry method using bovine serum albumin (BSA) as the standard (Lowry et al., 1951) and the results revealed that APF3 contained $2.7 \%$ protein whereas APF1 and APF2 were free of proteins. As described above, APF3 was proven to possess the highest antioxidant activity among the polysaccharide fractions, but it was still unclear whether the activity was associated with bound-protein in APF3. Therefore, the present study was also set up to investigate the effect of co-extracted proteins on LDH leakage and MDA formation in $t$-BHP-induced macrophages. In this experiment, the proteins in APF3 were digested with pronase and then the pronase was inactivated at $70^{\circ} \mathrm{C}$ for $15 \mathrm{~min}$, and the remaining polysaccharides were lyophilized after extensive dialysis. The results of protein and carbohydrate analysis as previously described (Yang et al., 2005; Lowry et al., 1951) showed that pronase-treated APF3 was free of proteins and no significant change in monosaccharide composition was observed. As shown in Fig. 6A and B, although the treatment with pronase did not destroy protective effect of APF3 against the oxidative damage in $t$-BHP-induced macrophage $(14.9 \pm$ $1.7 \%$ for $\mathrm{LDH}$ leakage and $1.35 \pm 0.52 \mathrm{nmol} / 10^{6}$ cells for MDA formation in the $t$-BHP control groups, respectively), pronase-treated APF3 remarkably increase the LDH leakage (Fig. 6A) and MDA levels (Fig. 6B) from the cultured macrophages in a dose-related manner compared to untreated APF3 $(p<0.05)$. From the result in Fig. 6A and B, it was evident that the bound-protein was closely associated with the antioxidant effect of APF3.

Examination for endotoxin contamination. A possible contamination of endotoxin LPS is always a matter of concern for the high molecular weight components isolated from plants because LPS is a strong activator of macrophages and 

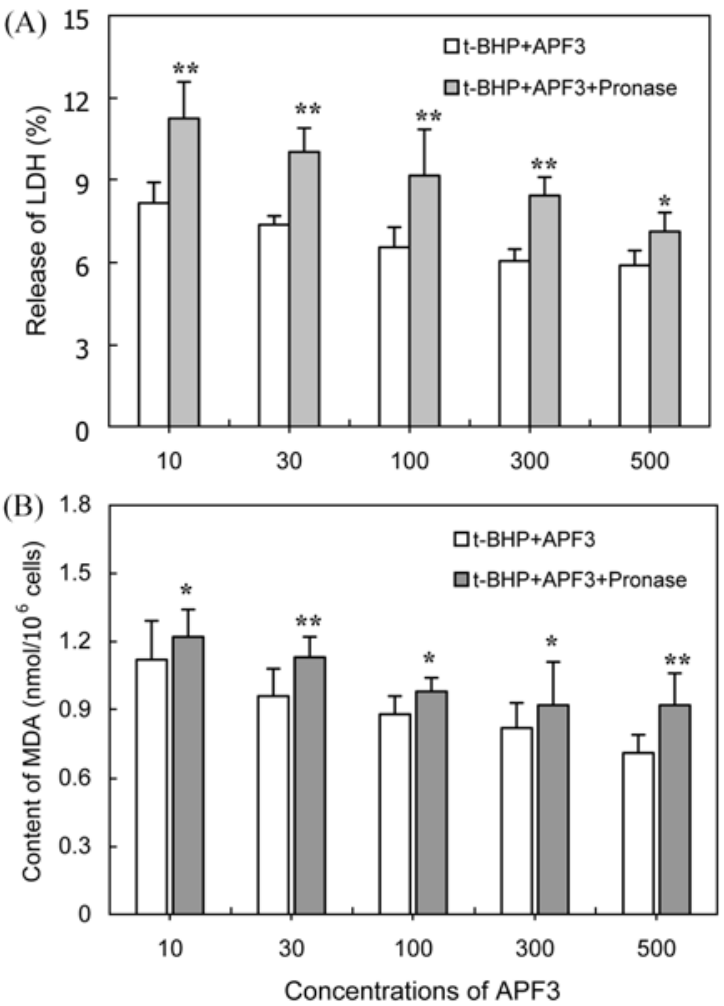

Fig. 6. Effects of bound-protein in APF3 on LDH release and MDA formation from $t$-BHP-injuried macrophages. The proteins in APF3 were digested with pronase and the remaining polysaccharides were lyophilized after extensive dialysis and added to the cultures of macrophages for $24 \mathrm{~h}$, then exposed to $0.2 \mathrm{mM} t$-BHP for another $24 \mathrm{~h}$. The LDH leakage and MDA contents were measured and date represent means $\pm \mathrm{SD}(\mathrm{n}=5) .{ }^{*} p<0.05,{ }^{* *} p<0.01$, compared to groups untreated with pronase.

is contaminated in many plant materials. For this reason, we examined the effect of polymyxin B (PMB) on APF3-induced NO production. PMB is known to inhibit LPS activity by binding to the lipid A moiety (Morrison and Jacobs, 1976). As shown in Fig. 7, the PMB treatment did not affect APF3 or $t$ BHP-induced NO generation, while it significantly abolished NO production in LPS-induced macrophages $(p<0.01)$. These results demonstrate that the polysaccharide fraction was not contained with LPS and the active component of APFs was appeared to be polysaccharides.

\section{Discussion}

ROS are frequently generated in aerobic bodies and scavenged by antioxidants. Various anti-oxidative defense systems in organism can effectively eliminate oxidative damages induced by oxidative stress under normal physiological conditions, and once the intensity of oxidative stress exceeds the threshold of antioxidant defense, a series of oxidative damages would be induced and further result in the pathological change (Wu et

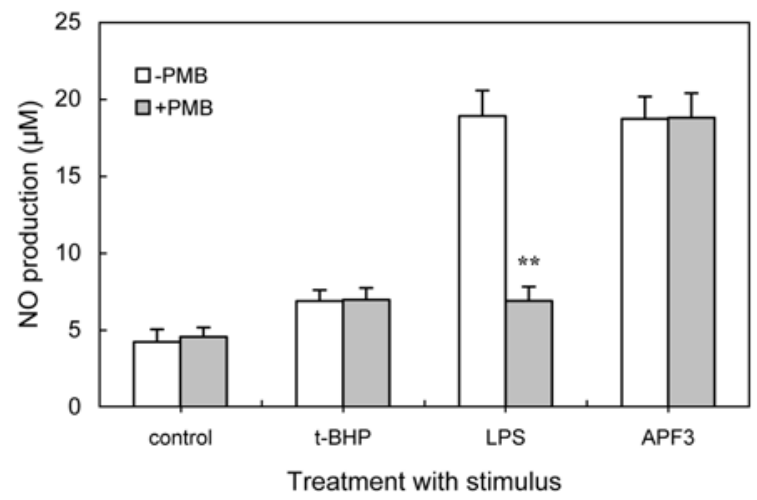

Fig. 7. Effects of polymyxin $\mathrm{B}(\mathrm{PMB})$ treatment on NO release from macrophages. APF3 $(300 \mu \mathrm{g} / \mathrm{ml})$, LPS $(5 \mu \mathrm{g} / \mathrm{ml})$, or $t$-BHP $(0.2 \mathrm{mM})$ stimuli were pre-incubated with PMB $(1000 \mathrm{U} / \mathrm{ml})$ for $1 \mathrm{~h}$, and then used to activate macrophages for $24 \mathrm{~h}$. NO generation was determined from the culture supernatant using Griess reagent. Significance was determined using the Student's $t$-test versus the corresponding group without PMB treatment $(* * p<0.01)$.

al., 2006). This process is especially evident in the immune cells, which exert their bio-functions through free radicals and suffer senescent deterioration probably linked to oxygen stress. Conversely, antioxidant supplementation has been proved to preserve an adequate function of immune cells against homeostatic disturbances caused by oxidative stress. Therefore, the protection of immune system by dietary antioxidant may play an important role in preserving the immune function and achieving healthy aging (Chen et al., 2003; Han et al., 2005).

The polysaccharide isolated from Radix A. sinensis is one of the major active ingredients and their immunomodulatory activity has been elucidated (Sun et al., 2005). In this study, the cytotoxicity was measured by MTT and LDH leakage, and the oxidative damage was estimated by measuring MDA contents, GSH levels and SOD activities. Our data demonstrated that APFs could protect cultured murine peritoneal macrophages against $t$-BHP-mediated oxidative injury, indicating APFs had strong antioxidant ability. Cells are often equipped with several antioxidants for the prevention of free radical damage and SOD, along with other non-enzymatic antioxidants such as GSH, serve as a detoxifying system to prevent cell damage caused by ROS and play a pivotal role. In addition, NO is a key effector molecule in macrophages and acts as an antioxidant by eliminating superoxide, and on the other hand, its reaction product $\left(\mathrm{ONOO}^{-}\right)$is an attractive candidate for cytotoxicity (Wu and Huang, 2006). In our experiments, APFs could inhibit $t$-BHP-mediated oxidative injury through a reduction in MDA formation and an increase in SOD activity and GSH content to maintain membrane integrity. In addition, APFs enhanced NO release in the low concentration of $t$ BHP-stimulated macrophages, where increasing NO contributed to the killing of microorganisms and tumor cells and mediated a variety of biological functions as an intracellular messenger molecule. In contrast, APFs also exhibited an inhibitory effect 
on NO production in the high concentration of $t$-BHPstimulated macrophages to protect host cells from the harm resulting from the excess NO production (Palmer et al., 1998), and the inhibition of NO generation was also probably associated with extensive cell death under very high concentration of $t$ BHP.

In conclusion, there was a significant increase in lipid peroxidation when the macrophages were exposed to $t$-BHP and the polysaccharide fractions did exhibit expectedly strong protective effects against $t$-BHP-induced injury in cultured murine peritoneal macrophages.

Acknowledgments This work was supported by the science and research foundations of Shaanxi Province and Shaanxi Normal University, China (No. SF200224; No:2002K10-G2).

\section{References}

Abe, K., McKibbin, J. M. and Hakomori, S. (1983) The monoclonal antibody directed to difucosylated type 2 chain (Fuc alpha 1 leads to $2 \mathrm{Ga} 1$ beta 1 leads to $4[\mathrm{Fuc}$ alpha 1 leads to 3]G1cNAc; Y determinant). J. Biol. Chem. 258, 11793-11797.

Bonnefont-Rousselot, D., Bastard, J. P., Jaudon, M. C. and Delattre, J. (2000) Consequences of the diabetic status on the oxidant/ antioxidant balance. Diabetes Metab. 26, 163-176.

Carmichael, J., DeGraff, W. G., Gazdar, A. F., Minna, J. D. and Mitchell, J. B. (1987) Evaluation of a tetrazolium-based semiautomatic colorimetric assay: assessment of chemosensitivity testing. Cancer Res. 47, 936-942.

Chen, A. S., Taguchi, T., Sakai, K., Kikuchi, K., Wang, M. W. and Miwa, I. (2003) Antioxidant activities of chitobiose and chitotriose. Biol. Pharm. Bull. 26, 1326-1330.

Deng, S., Chen, S. N., Yao, P., Nikolic, D., van Breemen, R. B., Bolton, J. L., Fong, H. H., Farnsworth, N. B. and Bolton, J. L. (2006) Serotonergic activity-guided phytochemical investigation of the roots of Angelica sinensis. J. Nat. Prod. 69, 536-541.

Dipti, K. P., Sharma, S. K., Sairam, M., Ilavazhagan, G., Sawhney, R. C. and Banerjee, P. K. (2006) Flavonoids protect U-937 macrophages against tert-butylhydroperoxide induced oxidative injury. Food Chem. Toxicol. 44, 1024-1030.

Fang, X. B., Jiang, B. and Wang, X. L. (2006) Purification and partial characterization of an acidic polysaccharide with complement fixing ability from the stems of Avicennia marina. J. Biochem. Mol. Biol. 39, 546-555.

Green, L. C., Wagner, D. A., Glogowski, J., Skipper, P. L., Wishnok, J. S. and Tannenbaum, S. R. (1982) Analysis of nitrate in biological fluids. Anal. Biochem. 126, 131-138.

Han, S. H., Espinoza, L. A., Liao, H. L., Boulares, A. H. and Smulson, M. E. (2004) Protection by antioxidants against toxicity and apoptosis induced by the sulphur mustard analog 2chloroethylethyl sulphide (CEES) in Jurkat T cells and normal human lymphocytes. Brit. J. Pharmacol. 141, 795-802.

Han, Y., zhao, L., Yu, Z., Feng, J. and Yu, Q. (2005) Role of mannose receptor in oligochitosan-mediated stimulation of macrophage function. Int. Immunopharmacol. 5, 1533-1542.

Jeong, S. C., Jeong, Y. T., Yang, B. K. and Song, C. H. (2006) Chemical characteristics and immuno-stimulating properties of biopolymers extracted from Acanthopanax sessiliflorus. J. Biochem. Mol. Biol. 39, 84-90.

Kim, G. Y., Choi, G. S., Lee, S. H. and Park, Y. M. (2004) Acidic polysaccharide isolated from Phellinus linteus enhances through the up-regulation of nitric oxide and tumor necrosis factor- $\alpha$ from peritoneal macrophages. J. Ethnopharmacol. 95, 69-76.

Kim, H. H., Bang, S. S., Choi, J. S., Han, H., Kim, I. H. (2005) Involvement of $\mathrm{PKC}$ and ROS in the cytotoxic mechanism of anti-leukemic decursin and its derivatives and their structureactivity relationship in human K562 erythroleukemia and U937 myeloleukemia cells. Cancer Lett. 223, 191-201.

Kim, N. H. and Kang, J. H. (2006) Oxidative damage of DNA induced by the cytochrome c and hydrogen peroxide system. $J$. Biochem. Mol. Biol. 39, 452-456.

Lao, S. C., Li, S. P., Kan, K. K. W., Li, P., Wan, J. B., Wang, Y. T., Dong, T. T. X. and Tsim, K.W. K. (2004) Identification and quantification of 13 components in Angelica sinensis (Danggui) by gas chromatography-mass spectrometry coupled with pressurized liquid extraction. Anal. Chim. Acta 526, 131-137.

Lowry, O. H., Rosebrough, N. J., Farr, A. L. and Randall, R. J. (1951) Protein measurement with the Folin phenol reagent. $J$. Biol. Chem. 193, 265-275.

Lu, G. H., Chan, K., Chan, C. L., Leung, K., Jiang, Z. H., Zhao, Z. Z. (2004) Quantification of ligustilides in the roots of Angelica sinensis and related umbelliferous medicinal plants by highperformance liquid chromatography and liquid chromato-graphymass spectrometry. J. Chromatogr. A 1046, 101-107.

MacMicking, J., Xie, Q. W. and Nathan, C. (1997) Nitric oxide and macrophage function. Annu. Rev. Immunol. 15, 323-350.

Majeti, N. V. and Ravi Kumar, R. (2000) A review of chitin and chitosan. React. Funct. Polym. 46, 1-27.

Mansour, H. H., Hafez, H. F. and Fahmy, N. M. (2006) Silymarin modulates cisplatin-induced oxidative stress and hepatotoxicity in rats. J. Biochem. Mol. Biol. 39, 656-661.

Mi, Y. L., Zhang, C. Q. (2005) Protective effect of quercetin on aroclor 1254-induced oxidative damage in cultured chicken sermatogonial cells. Toxicol. Sci. 88, 545-550.

Morrison, D. C. and Jacobs, D. M. (1976) Binding of polymyxin B to the lipid A portion of bacterial lipopolysaccharides. Immunochemistry 13, 813-818.

Mossman, T. (1983) Rapid colorimetric assay for cellular growth and survival: application to proliferation and cytotoxicity assays. $J$. Immunol. Methods 65, 55-63.

Palmer, R. M. J., Ashton, D. S. and Moncada, S. (1988). Vascular endothelial cells synthesize nitric oxide from L-arginine. Nature 333, 664-666.

Rojas, A., Padron, J., Caveda, L., Palacios, M. and Moncada, S. (1993) Role of nitric oxide pathway in the protection against lethal endotoxemia by low dose of lipopolysaccharides. Biochem. Biophys. Res. Commun. 191, 441-446.

Shahidi, F. and Synowiecki, J. (1991) Isolation and characterization of nutrients and value-added products from snow crab (Chinoecetes opilio) and shrimp (Pandalus borealis) processing discards. $J$. Agr. Food Chem. 39, 1527-1532.

Srivastava, R. and Kulshreshtha, D. K. (1989) Bioactive polysaccharide from plants. Phytochemistry 28, 2877-2883.

Sun, Y. L., Tang, J., Gu, X. H. and Li, D. Y. (2005) Water-soluble polysaccharides from Angelica sinensis (Oliv.) Diels: Preparation, characterization and bioactivity. Int. J. Biol. Macromol. 36, 283289. 
Sun, Y., Oberley, L. W. and Ying, L. (1988). A simple method for clinical assay of superoxide dismutase. Clin. Chem. 34, 497-500.

Uchiyama, M. and Mihara, M. (1978) Determination of malonaldehyde precursor in tissues by thiobarbituric acid test. Anal. Biochem. 86, 271-278.

Van den Berg, J. J., Op den Kamp, J. A., Lubin, B. H., Roelofsen, B. and Kuypers, F. A. (1992) Kinetics and site specificity of hydroperoxide-induced oxidative damage in red blood cells. Free Radical Bio. Med. 12, 487-498.

Wu, Q. Z. and Huang, K. X. (2006) Protective effect of ebselen on cytotoxicity induced by cholestane-3 beta, 5 alpha, 6 beta-triol in ECV-304 cells. Biochim. et Biophys. Acta 1761, 350-359.
Wu, J. H., Xu, C., Shan, C. Y. and Tan, R. X. (2006) Antioxidant properties and PC12 cell protective effects of APS-1, a polysaccharide from Aloe vera var. chinensis. Life Sci. 78, 622630 .

Yang, X. B., Zhao, Y., Wang, Q. W., Wang, H. F. and Mei, Q. B. (2005). Analysis of the monosaccharide components of Angelica polysaccharides by high performance liquid chromatography. Anal. Sci. 21, 1177-1180.

Zhao, K. J., Dong, T. T. X., Tu, P. F., Song, Z. H., Lo, C. K. and Tsim, K. W. K. (2003) Molecular genetic and chemical assessment of radix Angelica (Danggui) in China. J. Agr. Food Chem. 51, 2576-2583. 